\title{
Zu häufiges Händewaschen hat häufig Folgen
}

\author{
Im Gesundheitsbereich tätige Personen waschen \\ sich nach wie vor zu oft die Hände und leiden auch \\ deutlich häufiger an Handekzemen. Regelmäßiges \\ Händedesinfizieren dagegen scheint nach einer \\ aktuellen dänische Studie Handekzeme nicht \\ wesentlich zu begünstigen.
}

D ermatologen der Universitäten Kopenhagen und Roskilde hatten 3.181 Ärzten, Krankenschwestern und -pflegern sowie klinisch-technischen Angestellten von Universitätskliniken einen Fragebogen zu berufsbedingten Hauterkrankungen zugesendet. 71\% der Studienteilnehmer schickten den Bogen ausgefüllt zurück. Aus den Angaben ermittelten die Forscher Risikofaktoren für die Entwicklung von Handekzemen in dieser Berufsgruppe.

Die Auswertungen zeigten, dass knapp jeder sechste an einem Handekzem litt. Die Teilnehmer an der Befragung, die ein Handekzem hatten, wuschen sich ihre Hände signifikant häufiger als Teilnehmer ohne entsprechenden Befund. So berichteten $52 \%$ der Befragten mit einem Handekzem, sich die Hände mehr als zehnmal pro Tag zu waschen; $19 \%$ hielten ihre Hände sogar mehr als 20-mal unters laufende Wasser. In der Gruppe ohne Ekzeme lagen die ensprechenden Quoten mit $43 \%$ bzw. $12 \%$ jeweils signifikant niedriger.

Kein Unterschied zeigte sich dagegen beim Gebrauch von Desinfektionsmitteln: Etwa die Hälfte der Studienteilnehmer - mit und ohne Ekzem - gab an, öfter als 20-mal pro Tag auf den Desinfektionsmittelspender zu drücken. Auch wer Kinder unter vier Jahren zu betreuen hatte, trug ein signifikant höheres Risiko für Ekzeme. Statistisch auffällig war zudem, dass die Prävalenz von Handekzemen bei den Befragten niedriger lag, die während ihrer Arbeitszeit regelmäßig Feuchtigkeitscremes benutzten.

Fazit: Die dänischen Dermatologen fordern verhaltensändernde Maßnahmen - und zwar nicht nur für die Arbeitszeit. Denn die an Handekzemen Erkrankten wuschen sich ihre Hände auch zu Hause häufiger als Studienteilnehmer ohne Ekzeme. Besonderes Augenmerk sollte dabei jenen Personen gelten, die kleine Kinder zu Hause haben.

Handekzeme sind bei Angehörigen medizinischer Berufe mit einer Prävalenz von bis zu $30 \%$ etwa dreimal häufiger als in der Allgemeinpopulation. Umgekehrt arbeiten rund ein Fünftel aller Patienten mit berufsbedingten Handekzemen im Gesundheitsbereich. Die Kosten, die durch die Therapie von Handekzemen entstehen, sind erheblich. In einer Studie des Competenzzentrums Versorgungsforschung in der Dermatologie an der Universität Hamburg vom vergangenen Jahr wurden Zahlen genannt, die je nach Therapieform zwischen 1.044 und 8.407 Euro pro Patient und Jahr lagen. Dr. Robert Bublak

Ibler KS et al. Exposures related to hand eczema: a study of healthcare workers. Contact Dermatitis 2012; 66: 247-53 